\title{
Tracking a Minimum Bounding Rectangle based on Extreme Value Theory
}

\author{
Marcus Baum and Uwe D. Hanebeck
}

\begin{abstract}
In this paper, a novel Bayesian estimator for the minimum bounding axis-aligned rectangle of a point set based on noisy measurements is derived. Each given measurement stems from an unknown point and is corrupted with additive Gaussian noise. Extreme value theory is applied in order to derive a linear measurement equation for the problem. The new estimator is applied to the problem of group target and extended object tracking. Instead of estimating each single group member or point feature explicitly, the basic idea is to track a summarizing shape, namely the minimum bounding rectangle, of the group. Simulation results demonstrate the feasibility of the estimator.
\end{abstract}

\section{INTRODUCTION}

Minimum bounding rectangles are frequently used in many application areas like image processing, geography, and tracking. This paper is concerned with the problem of estimating the minimum bounding axis-aligned rectangle of a set of points. However, the points, which we also call measurement sources, are not given directly. Only measurements corrupted with Gaussian noise are available. Furthermore, it is not known from which measurement source a particular measurement stems.

The contributions of the paper are the following: We derive a novel recursive Bayesian estimator [1] for the minimum axis-aligned bounding rectangle given noisy position measurements. In order to derive the estimator, we employ techniques from extreme value theory, which deals with the probability of the minimum or maximum of random experiments. The resulting measurement equation is linear, so that the well-known Kalman filter can be used for inference.

Finally, we apply the proposed estimator to the problem of tracking a group of point targets [2] (see Fig. 1). The basic approach followed here is to track the minimum bounding rectangle of the group, instead of each single group member. This is suitable in cases where data association is too difficult or one is simply not interested in the exact position of the individual group members. For example, data association may become hard, when the group members are closely spaced, i.e., their validation gates overlap. For a large number of targets, e.g., more than 10, data association algorithms then may even become computationally intractable.

A similar problem occurs when tracking an extended target object, where several point features, i.e., measurement sources, on the target surface may cause measurements. In this case, the proposed method can be used to track the

M. Baum and U. D. Hanebeck are with the Intelligent Sensor-ActuatorSystems Laboratory (ISAS), Institute for Anthropomatics, Karlsruhe Institute of Technology (KIT), Germany. marcus.baumekit.edu, uwe. hanebeck@ieee.org
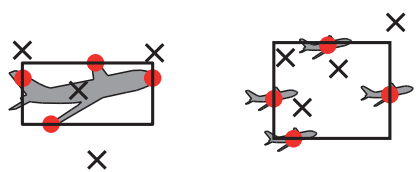

$\begin{aligned} & =\text { Measurement source } \\ X & =\text { Measurement }\end{aligned}$

Fig. 1: Extended object and group of targets.

minimum bounding rectangle of the shape of the extended object. Such tracking problems arise for instance in air surveillance, where aircraft are tracked with high-resolution radar devices (see Fig. 1). The estimator proposed in this paper is suitable if the minimum bounding rectangle of the measurement sources coincides with the minimum bounding rectangle of the target (see Fig. 1). It is important to note that the application area for summarizing shape estimation in tracking is different from Spatial Distribution Models [3] or Random Hypersurface Models [4]. These models are suitable for a rather diffuse measurement generation process, e.g., scenarios with low detection probability, partly unresolved targets, and high measurement noise.

The remainder of this paper is structured as follows: After a brief overview of related methods in Section II, we give a detailed problem formulation in Section III. Then, we introduce some basic results from extreme value theory (see Section IV), which are employed for deriving the novel estimator for the minimum bounding rectangle (see Section V). The proposed estimator is then evaluated in Section VI. Finally, conclusions and an outlook to future work are given in Section VII.

\section{RELATED WORK}

In this section, we give a short overview of related methods to the considered problem. Because the measurement sources for which the smallest enclosing rectangle is to be estimated are unkwown (and should not be estimated explicitely), it is necassary to make proper (implicit) assumptions on the location of the measurement sources. One statistical approach for the problem of estimating rectangles is to assume that each measurement source is an independent random draw from a uniform distribution on the true rectangle. Such a distribution is also called spatial distribution [3], [5]. This approach suffers from the disadvantage that in real-world applications, the measurement sources are in fact not uniformly distributed on the rectangle. Then, a spatial distribution model may provide poor estimation results. Furthermore, there are no closedform expressions for a Bayesian solution with a uniform spatial distribution on a rectangle available. Non-Bayesian 


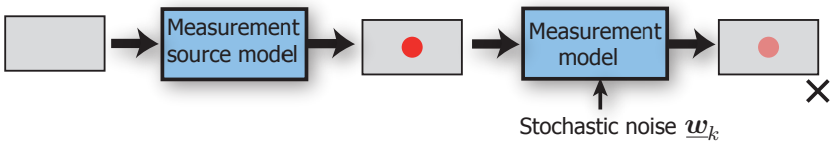

(a) Independent generation of each single measurement source. Examples: Random Hypersurface Models, Spatial Distribution Models.

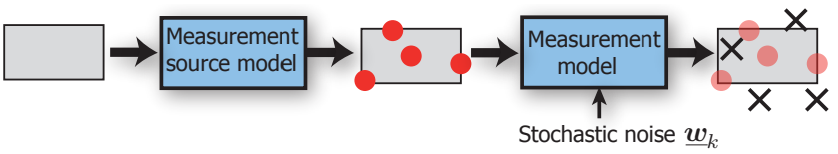

(b) Batch generation of all measurement sources. The true shape is the smallest enclosing shape of the measurement sources.

$=$ Measurement source

$\mathrm{X}=$ Measurement

$=$ True Shape

Fig. 2: Different models for generating measurement sources at a particular time step.

solutions to this problems have also been considered in a context different from tracking, for instance in [6].

In [4], so-called Random Hypersurface Models are introduced for the purpose of tracking extended targets. This approach assumes that each measurement source is an element of a hypersurface generated from a random draw of a onedimensional probability distribution. In fact, this approach could also be used to model a rectangle. However, also a Random Hypersurface Model imposes statistical assumptions on the measurement sources and no closed-form expressions are currently available for rectangles.

A further related approach for tracking rectangular-shaped extended target objects was introduced in [7]. There, it is only assumed that the measurement sources lie on the target surface. No (statistical) assumptions about the measurement sources are made. If there is no measurement noise, the problem can be formulated as a set-theoretic estimation problem. Stochastic measurement noise then requires a combined settheoretic and stochastic estimator. With this approach it is not possible to estimate the size of the target extent only with position measurements. In [7], it is necessary to assume that the number of measurements, which are received at a particular time step depends on the size of the extended object in order to estimate the size of minimum bounding rectangle. This is a quite restrictive assumption, which, however, is often fulfilled in target tracking applications. The approach presented in this paper does not have this restriction.

Apart from the above approaches, which implicitly model measurement sources, there also exist approaches that explicitly model each single measurement sources on the target extent [8], [9], [10]. These approaches require data association and are thus computationally expensive. Furthermore, they require dynamic models for the measurement sources, which may not be available.

\section{PROBLEM FORMULATION}

We treat the problem of tracking the parameters of the axis-aligned minimum bounding rectangle of a finite set of
$N$-dimensional points based on measurements corrupted with additive Gaussian noise. At each time step $k$, a finite set of measurements $\mathcal{Z}_{k}:=\left\{\underline{\hat{z}}_{k, l}\right\}_{l=1}^{n_{k}}$ becomes available. Each individual measurement $\underline{\hat{z}}_{k, l} \in \mathbb{R}^{N}$ is the noisy observation of a point $\underline{\tilde{z}}_{k, l} \in \mathbb{R}^{N}$, named measurement source, i.e.,

$$
\underline{\hat{z}}_{k, l}=\underline{\tilde{z}}_{k, l}+\underline{\boldsymbol{w}}_{k, l},
$$

where $\underline{\boldsymbol{w}}_{k, l}$ denotes additive white Gaussian noise ${ }^{1}$ with diagonal covariance matrix $\operatorname{diag}\left(\left[\sigma_{1}, \ldots, \sigma_{N}\right]\right)$. The location of the measurement source $\underline{\tilde{z}}_{k, l}$ is totally unknown. The goal is to estimate the parameters of the axis-aligned minimum bounding rectangle of $\left\{\underline{\tilde{z}}_{k, l}\right\}_{l=1}^{n_{k}}$.

Since we focus on tracking applications, the parameters of the minimum bounding rectangle may evolve over time. Its temporal evolution is modeled by means of a stochastic motion model. Note that no dynamic models for the measurement sources itself are given.

In this paper, we seek a Bayesian estimator [1] for the minimum bounding rectangle, which is a recursive update scheme for a probability distribution over the unknown state according to Bayes' rule .

REMARK 1 The above problem formulation is different from Spatial Distribution Models [3] and Random Hypersurface Models [4], which were introduced in the context of extended object tracking. There, each single measurement source is generated independently according to a stochastic process. As a consequence, these models allow for receiving a single measurement at a particular time step from an extended object. Here, a single measurement per time step would immediately yield a point since the minimum bounding rectangle of a point is a point. These two different generative models for the measurements are illustrated in Fig. 2.

REMARK 2 The above problem is fundamentally different from statistical shape fitting [11], [12], [13]. In statistical shape fitting, one deals with estimating a shape, like a circle, from given noisy measurements. However, the big difference is that in shape fitting, the measurement sources all stem from the border of the shape. Here, the measurement sources may also lie in the inner of the shape, i.e., the rectangle.

\section{EXTREME VALUE THEORY}

In this section, a brief introduction to extreme value theory is given. Extreme value theory [14] is a branch of statistics that deals with extreme values such as minima and maxima of random experiments. The application area of extreme value theory is quite broad. Just to mention a few, it has been applied for (financial) risk management [15], flood prediction, engineering and, insurance assessment [16].

In probability theory, the Central Limit Theorem states that the mean of a sequence of independent identically distributed random variables approaches a normal distribution (under certain assumptions). Similarly, it can be shown that the extreme value of a sequence of independent identically distributed random variables approaches a limiting distribution.

\footnotetext{
${ }^{1}$ All random variables are printed bold face in this paper.
} 
Let $\boldsymbol{x}_{1}, \ldots, \boldsymbol{x}_{n}$ be a sequence of independent identically distributed random variables with common probability distribution function $F(x)$. The maximal element of this sequence is given by the random variable

$$
\boldsymbol{M}_{n}:=\max \left\{\boldsymbol{x}_{1}, \ldots, \boldsymbol{x}_{n}\right\} .
$$

The so-called Fisher-Tippet-Gnedenko theorem or extreme value theorem, which is a main result in extreme value theory, says that with normalizing sequences $\left\{a_{n}\right\}$ and $\left\{b_{n}\right\}$, the probability distribution of

$$
\frac{M_{n}-a_{n}}{b_{n}}
$$

approaches the generalized extreme value (GEV) distribution.

$$
G(x ; \alpha, \beta, \xi)=\exp \left\{-\left[1+\xi\left(\frac{x-\alpha}{\beta}\right)\right]^{-1 / \xi}\right\}
$$

with $1+\xi(x-\mu) / \sigma>0$, where $\mu \in \mathbb{R}$ is the location parameter, $\sigma>0$ the scale parameter and $\xi \in \mathbb{R}$ the shape parameter. The shape parameter $\xi$ reflects the weight of the tail of the distribution $F(\cdot)$ and yields to the following special cases:

- For $\xi \rightarrow 0$, the so-called Gumble or Type I extreme value distribution is obtained

$$
G^{1}(x ; \alpha, \beta)=\exp \{-\exp \{(\alpha-x) / \beta\}\} .
$$

- $\xi>0$ results in the the Fréchet or Type II extreme value distribution.

- For $\xi<0$, the Reversed Weibull or Type III extreme value distribution is obtained.

Probability distributions whose tails decrease exponentially yield a Gumble distribution. This class of probability distributions for instance include the exponential distribution and the normal distribution. In this paper, we only deal with normally distributed random variable, such that we only consider Gumble distributions in the following.

The distribution of $\boldsymbol{M}_{n}$, can be approximated with a Gumble distribution $G^{1}\left(x ; \alpha_{n}, \beta_{n}\right)$ with

$$
\begin{aligned}
\alpha_{n} & :=F^{-1}\left(1-\frac{1}{n e}\right)-\beta_{n}, \\
\beta_{n} & :=F^{-1}\left(1-\frac{1}{n}\right) .
\end{aligned}
$$

The precision of this approximation is illustrated in Fig. 3. Furthermore, it can be seen that the Gumble distribution is similar to a normal distribution.

The first two moments of a Gumble distribution $G^{1}\left(x ; \alpha_{n}, \beta_{n}\right)$ are

$$
\begin{aligned}
\mu & =\alpha_{n}+\gamma \beta_{n} \\
\sigma & =\alpha_{n} \pi \sqrt{6}
\end{aligned}
$$

where $\gamma \approx 0.577215$ is the Euler-Mascheroni constant.

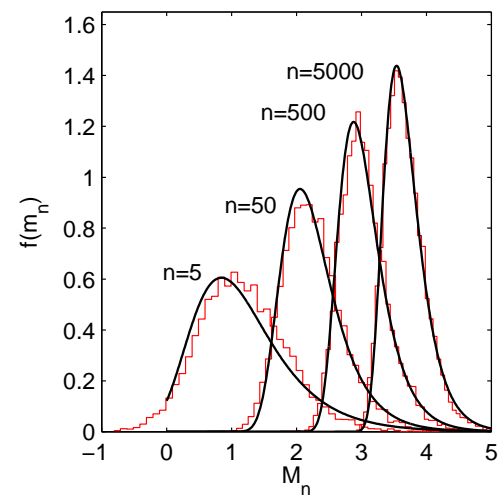

Fig. 3: Approximation of the maximum $\boldsymbol{M}_{n}$ of $n$ Gaussian random variables with a Gumble distribution (black) and histogram (red) for 10000 samples of $\boldsymbol{M}_{n}$.

\section{BAYESIAN ESTIMATION OF THE MINIMAL BOUNDING RECTANGLE}

In this section, we introduce a recursive Bayesian estimator for the minimum bounding rectangle according to the problem description in Section III. An axis-aligned rectangle in $N$-dimensional space is represented with the extreme values (the minimum and maximum) on each axis. Hence, the uncertainty of the minimum bounding rectangle at time step $k$ is represented with the random vector

$$
\underline{\boldsymbol{x}}_{k}=\left[\boldsymbol{l}_{k}^{1}, \boldsymbol{u}_{k}^{1}, \ldots, \boldsymbol{l}_{k}^{N}, \boldsymbol{u}_{k}^{N}\right]^{T}
$$

where for all $1 \leq i \leq N$

- $l_{k}^{i}$ represents the lower bound of the rectangle on the $i$-th axis, and

- $\boldsymbol{u}_{k}^{i}$ represents the upper bound of the rectangle on the $i$-th axis.

The parameters of the true rectangle to be estimated at time step $k$ are given by

$$
\tilde{x}_{k}=\left[\begin{array}{c}
\tilde{l}_{k}^{1} \\
\tilde{u}_{k}^{1} \\
\vdots \\
\tilde{l}_{k}^{N} \\
\tilde{u}_{k}^{N}
\end{array}\right]=\left[\begin{array}{c}
\min _{1 \leq j \leq n_{k}}\left\{\tilde{z}_{k, j}^{i}\right\} \\
\max _{1 \leq j \leq n_{k}}\left\{\tilde{z}_{k, j}^{i}\right\} \\
\vdots \\
\min _{1 \leq j \leq n_{k}}\left\{\tilde{z}_{k, j}^{i}\right\} \\
\max _{1 \leq j \leq n_{k}}\left\{\tilde{z}_{k, j}^{i}\right\}
\end{array}\right] .
$$

The goal is to derive an estimator that recursively computes the probability density of $\underline{\boldsymbol{x}}_{k}$ given the accumulated measurements $\mathcal{Z}_{1: k}:=\left\{\mathcal{Z}_{1}, \ldots, \overline{\mathcal{Z}}_{k}\right\}$

$$
p\left(\underline{x}_{k} \mid \mathcal{Z}_{1: k}\right) \text {. }
$$

We assume that this probability density is Gaussian, i.e., $p\left(\underline{x}_{k} \mid \mathcal{Z}_{1: k}\right)=\mathcal{N}\left(\underline{x}_{k}-\mu_{k}^{e}, \mathbf{C}_{k}^{e}\right)$.

For this purpose, we first derive a linear measurement equation that relates the state $\underline{\boldsymbol{x}}_{k}$ to the measurements $\mathcal{Z}_{k}$ The Kalman filter [17] equations then provide expression for the optimal state update. 


\section{A. Derivation of the Measurement Equation}

First, we restrict to a particular axis $i$ and the upper bound $\tilde{u}_{k}^{i}$ of the minimum bounding rectangle. In order to estimate $\tilde{u}_{k}^{i}$, we first make the following decisive assumption: The $i$-th coordinate of a subset of the measurement sources coincides with $\tilde{u}_{k}^{i}$, i.e.,

$$
\tilde{z}_{k, j}^{i}=\tilde{u}_{k}^{x} \text { for all } j \in \mathcal{B}_{k}^{u, i},
$$

where $\mathcal{B}_{k}^{u, i}$ is a set of indices of measurements. The other measurement sources are assumed to be far away from $\tilde{u}_{k}^{i}$ so that they do not influence the maximum distribution. Then, obviously, the following holds:

$$
\tilde{u}_{k}^{i}=\max _{i \in \mathcal{B}_{k}^{u, i}}\left\{\tilde{z}_{k}^{i}\right\} .
$$

If the number of elements $\left|\mathcal{B}_{k}^{u, i}\right|$ in $\mathcal{B}_{k}^{u, i}$ is known, a Bayesian estimator for $\tilde{l}_{k}^{x}$ can be derived. Because of the above assumption $\boldsymbol{z}_{k, j}^{i}$ are independently identically-distributed for $j \in \mathcal{B}_{k}^{u, i}$. Hence, the probability distribution of

$$
\max _{j \in \mathcal{B}_{k}^{u, i}}\left\{\boldsymbol{z}_{k, j}^{i}\right\}-\tilde{u}_{k}^{i}:=\boldsymbol{w}_{k}^{i, u}
$$

is approximately Gumble distributed $G^{1}\left(x ; a_{k}^{u, i}, b_{k}^{u, i}\right)$ with

$$
\begin{aligned}
a_{k}^{u, i} & :=\Phi^{-1}\left(1-\frac{1}{\left|\mathcal{B}_{k}^{u, i}\right| e}\right)-b_{k}^{u, i} \\
b_{k}^{u, i} & :=\Phi^{-1}\left(1-\frac{1}{\left|\mathcal{B}_{k}^{u, i}\right|}\right)
\end{aligned}
$$

where $\left|\mathcal{B}_{k}^{u, i}\right|$ denotes the number of elements in $\mathcal{B}_{k}^{u, i}$ and $\Phi^{-1}$ is the inverse cumulative distribution function of a Gaussian distribution with zero mean and variance $\sigma_{i}$.

According to Section IV, the Gumble distribution can be approximated with a Gaussian distribution by means of analytic moment matching. A reformulation of (2) yields the linear measurement equation

$$
\hat{u}_{k}^{i}=\boldsymbol{u}_{k}^{i}+\boldsymbol{w}_{k}^{u, i},
$$

where $\boldsymbol{w}_{k}^{u, i}$ is approximated with a Gaussian with mean $a_{k}^{u, i}+\gamma b_{k}^{u, i}$ and variance $a_{k}^{u, i} \pi \sqrt{6}$ and virtual measurement

$$
\hat{u}_{k}^{i}:=\max _{0 \leq j \leq n_{k}}\left\{\underline{\hat{z}}_{k, j}^{i}\right\} .
$$

Unfortunately, the number of elements in $\mathcal{B}_{k}^{u, i}$ is unknown. We therefore propose a simple but effective heuristic to determine it:

Let $\boldsymbol{r}_{k}^{u, i}$ be the number of measurements $\underline{\hat{z}}_{k, u}$ with $\underline{\hat{z}}_{k, j}^{i}>$ $\tilde{u}_{k}^{x}$. Then, the discrete random variable $\boldsymbol{r}_{k}^{u, i}$ is Binomial distributed according to

$$
p\left(\boldsymbol{r}_{k}^{u, i}=r\right)=\left(\begin{array}{c}
\left|\mathcal{B}_{k}^{u, i}\right| \\
r
\end{array}\right) 0.5^{r} .
$$

The expectation of $\boldsymbol{r}_{k}^{u, i}$ is $\mathrm{E}\left[\boldsymbol{r}_{k}^{u, i}\right]=0.5 \cdot\left|\mathcal{B}_{k}^{u, i}\right|$. Hence, a proper approximation for $\left|\mathcal{B}_{k}^{u, i}\right|$ is given by

$$
\left|\mathcal{B}_{k}^{u, i}\right| \approx 2 \cdot \hat{r}_{k}^{u, i}
$$

where $\hat{r}_{k}^{u, i}:=\left|\left\{j \mid \underline{\underline{z}}_{k, j}^{i}>\hat{u}_{k}^{i}\right\}\right|$ and $\hat{u}_{k}^{i}$ is the current estimate for $\tilde{u}_{k}^{i}$. The estimate for $\left|\mathcal{B}_{k}^{u, i}\right|$ can also be averaged over several time steps in order to obtain more robust values. However, then temporal changes of $\left|\mathcal{B}_{k}^{u, i}\right|$ are followed slower. For small $\left|\mathcal{B}_{k}^{u, i}\right|$, i.e., $\left|\mathcal{B}_{k}^{u, i}\right|<4$, it is important that $\left|\mathcal{B}_{k}^{u, i}\right|$ is estimated precisely. However, with an increasing $\left|\mathcal{B}_{k}^{u, i}\right|$, it becomes less and less important to estimate $\left|\mathcal{B}_{k}^{u, i}\right|$ precisely. This results from the fact that the maximum approaches the limiting distribution quite fast (see Fig. 3).

In the same manner as for the upper bound $\tilde{u}_{k}^{i}$, a Bayesian estimator can be constructed for the lower bound $\tilde{l}_{k}^{i}$. With the pseudo measurement

$$
\hat{u}_{k}^{i}:=\min _{0 \leq j \leq n_{k}}\left\{\underline{\underline{z}}_{k, j}^{i}\right\},
$$

the measurement equation becomes

$$
\hat{l}_{k}^{i}=\boldsymbol{l}_{k}^{i}+\boldsymbol{w}_{k}^{l, i},
$$

where $\boldsymbol{w}_{k}^{l, i}$ is approximated with a Gaussian distribution with mean $-\left(a_{k}^{i}+\gamma b_{k}^{i}\right)$ and variance $a_{k}^{l, i} \pi \sqrt{6}$.

Finally, we have to compose the above measurement equations to a single measurement equation for all axes and extreme values. Since the measurement noise on different axes is uncorrelated, we obtain

$$
\underline{\hat{y}}_{k}=\underline{\boldsymbol{x}}_{k}+\underline{\boldsymbol{w}}_{k}
$$

with measurement

$$
\underline{\hat{y}}_{k}:=\left[\hat{l}_{k}^{1}, \hat{u}_{k}^{1}, \cdots, \hat{l}_{k}^{N}, \hat{u}_{k}^{N}\right]^{T}
$$

and Gaussian noise term

$$
\underline{\boldsymbol{w}}_{k}=\left[\boldsymbol{w}_{k}^{l, 1}, \boldsymbol{w}_{k}^{l, 1}, \cdots, \boldsymbol{w}_{k}^{l, N}, \boldsymbol{w}_{k}^{l, N},\right]
$$

with mean

$$
\mu_{k}^{w}=\left[\begin{array}{c}
-\left(a_{k}^{1}+\gamma b_{k}^{1}\right) \\
a_{k}^{1}+\gamma b_{k}^{1} \\
\vdots \\
-\left(a_{k}^{N}+\gamma b_{k}^{N}\right) \\
a_{k}^{N}+\gamma b_{k}^{N}
\end{array}\right]
$$

and covariance matrix

$$
\mathbf{C}_{k}^{w}=\pi \sqrt{6} \cdot \operatorname{diag}\left(\left[b_{k}^{1}, b_{k}^{1}, \ldots, b_{k}^{N}, b_{k}^{N}\right]\right) .
$$

One underlying assumption of the above measurement equation is that the minimum and maximum on a particular axis are independent. This assumption is fulfilled if the measurement noise is not greater than the width of the rectangle. However, we observed that even if this is not the case, the correlation between the maximum and minimum distribution is negligible.

\section{B. Measurement Update Step}

If the predicted probability density for the parameters at time step $k$ is Gaussian, i.e.,

$$
p\left(\underline{x}_{k} \mid \mathcal{Z}_{1: k-1}\right)=\mathcal{N}\left(\underline{x}_{k}-\mu_{k}^{p}, \mathbf{C}_{k}^{p}\right),
$$

the updated estimate $p\left(\underline{x}_{k} \mid \mathcal{Z}_{1: k}\right)$ according to measurement model (3) is also Gaussian with mean $\mu_{k}^{e}$ and covariance $\mathbf{C}_{k}^{e}$ and results from the Kalman filter equations

$$
\begin{aligned}
\mu_{k}^{e} & =\mu_{k}^{p}+\mathbf{K}_{k}\left(\underline{y}_{k}-\mu_{k}^{p}\right), \\
\mathbf{C}_{k}^{e} & =\left(\mathbf{I}-\mathbf{K}_{k}\right) \mathbf{C}_{k}^{p},
\end{aligned}
$$


with Kalman gain

$$
\mathbf{K}_{k}=\mathbf{C}_{k}^{p}\left(\mathbf{C}_{k}^{p}+\mathbf{C}_{k}^{v}\right)^{-1}
$$

\section{Prediction Step}

The parameter vector $\underline{x}_{k}$ of the rectangle is assumed to evolve according to a known Markov model characterized by the conditional density function $p\left(\underline{x}_{k} \mid \underline{x}_{k-1}\right)$. Thus, the predicted probability density at time step $k$, i.e., $p\left(\underline{x}_{k} \mid \mathcal{Z}_{1: k-1}\right)$, results from the Chapman-Kolmogorov equation

$$
p\left(\underline{x}_{k} \mid \mathcal{Z}_{1: k-1}\right)=\int p\left(\underline{x}_{k} \mid \underline{x}_{k-1}\right) p\left(\underline{x}_{k-1} \mid \mathcal{Z}_{1: k-1}\right) d \underline{x}_{k-1} .
$$

If $p\left(\underline{x}_{k-1} \mid \mathcal{Z}_{1: k-1}\right)$ is Gaussian and a linear system equation

$$
\underline{\boldsymbol{x}}_{k}=\mathbf{B}_{k} \underline{\boldsymbol{x}}_{k-1}+\underline{\boldsymbol{v}}_{k}
$$

with white Gaussian noise $\underline{\boldsymbol{v}}_{k}$ is given, the prediction $p\left(\underline{x}_{k} \mid \mathcal{Z}_{1: k-1}\right)$ is also Gaussian. Its mean $\mu_{k}^{p}$ and covariance matrix $\mathbf{C}_{k}^{p}$ can be computed with the formulas of the Kalman filter prediction step

$$
\begin{aligned}
\mu_{k}^{p} & =\mathbf{B}_{k} \mu_{k-1}^{e}, \\
\mathbf{C}_{k}^{p} & =\mathbf{B}_{k}^{T} \mathbf{C}_{k-1}^{e} \mathbf{B}_{k}+\mathbf{C}_{k-1}^{v} . \\
& \text { VI. EVALUATION }
\end{aligned}
$$

\section{A. Fixed Set of Measurement Sources}

The first example shows the applicability of the presented estimator for estimating the minimum bounding box. For this purpose, a fixed set of measurement sources are arranged in a plane. At each time step, noisy measurements are received from all measurement sources. The Gaussian measurement noise has covariance matrix of $\operatorname{diag}([1,1])$. The prior for the rectangle parameters are given by a Gaussian distribution with mean $[-1,9,0,9]^{T}$ and covariance matrix $\operatorname{diag}(4,4,4,4)$. The estimation results for three differents sets of measurement sources are depicted in Fig. 4.

The estimation results are compared with a spatial distribution model [3], [5] that assumes the measurement sources to be uniformly distributed on the entire rectangle surface. This spatial distribution model leads to the measurement likelihood function

$$
p\left(\underline{\hat{z}}_{k, l} \mid \underline{x}_{k}\right):=\frac{1}{R\left(\underline{x}_{k}\right)} \int_{R\left(\underline{x}_{k}\right)} \mathcal{N}\left(\underline{z}-\underline{\hat{z}}_{k, l}, \mathbf{C}_{k}^{v}\right) d \underline{z},
$$

where $R\left(\underline{x}_{k}\right)$ denotes the area of the rectangle specified by the parameter vector $\underline{x}_{k}$. As no closed-form expressions for the measurement update with this likelihood exists, we applied the Gaussian Particle Filter [18] for state estimation. Actually, the new approach is computationally far mor attractive than the spatial distribution model, as the new approach results in a linear formulation of the problem. The spatial distribution model is not suitable for higher dimensions.

The depicted results in Fig. 4 are chosen such that the estimation results have been converged, i.e., the results do not change anymore in the subsequent time steps. The different estimation results of the two estimators result from the different assumptions on the measurement sources. As the received measurement only stem from a finite set of measurement sources, the assumption made by the spatial distribution model is not proper. However, the assumptions made by the new approach appear to justified in this examples.

\section{B. Group Target Tracking}

The next example shows the feasibility of the new approach for tracking a group of point targets. For this purpose, 18 point targets are arranged in fixed relative positions. At each time step, the center of the group moves along the vector $\underline{\hat{u}}_{k-1}^{g}+\underline{\boldsymbol{v}}_{k-1}^{g}$, where $\underline{\hat{u}}_{k-1}^{g}:=[12,0]^{T}$ and $\underline{\boldsymbol{v}}_{k-1}^{g}$ is zero mean Gaussian noise with covariance matrix $\operatorname{diag}(0.01,0.2)$. After the fourth time step, the target group starts to perform a $90^{\circ}$ turn, i.e., the group rotates. For the parameters of the minimum bounding rectangle, we employed the motion model $\underline{\boldsymbol{x}}_{k}=\underline{\boldsymbol{x}}_{k-1}+\underline{\hat{\hat{u}}}_{k-1}+\underline{\boldsymbol{v}}_{k-1}$, The system input $\underline{\hat{\hat{u}}}_{k-1}$ is assumed to be

$$
\left[\begin{array}{llll}
0 & 0 & 1 & 1 \\
1 & 1 & 0 & 0
\end{array}\right]^{T} \underline{\hat{u}}_{k-1}^{g}
$$

which translates the lower and upper bounds of the rectangle according to $\underline{\hat{u}}_{k-1}^{g}$. At each time step, a single position measurement is received from each individual target (the probability of detection is 1 ). The measurement noise is zeromean Gaussian with covariance $\operatorname{diag}([1,1])$. Moreover, we made use of a prior density for $\underline{\boldsymbol{x}}_{1}$ with covariance matrix $\operatorname{diag}(1,1,1,1)$.

In this scenario, tracking each single group member would be quite hard, because of the large number of targets and overlapping validation gates. The example run in Fig. 5 shows that the minimal bounding rectangle of the group is tracked quite well. In particular, the $90^{\circ}$ turn is detected. Note that in real-world applications, more realistic motion models are required. Since the estimator is linear, it is straightforward to implement more complex motion models.

REMARK 3 It is important to note that the application area for summarizing shape estimation in tracking is different from the application area of Spatial Distribution Models or Random Hypersurface Models. These models are suitable for scenarios with low detection probability, partly unresolved targets and high measurement noise. In such a scenario, tracking a summarizing shape, like the minimum bounding rectangle, can be senseless. This is due to the fact that the minimum bounding box of the measurement sources may not coincide with the minimum bounding box of the target.

\section{CONCLUSIONS AND FUTURE WORK}

In this work, we have derived a Bayesian estimator for the axis-aligned minimum bounding box of a set of points given measurements corrupted with Gaussian additive noise. For this purpose, we have constructed a linear measurement equation with the help of extreme value theory. The applicability of the estimator has been demonstrated by means of a group target tracking example.

The presented estimator requires the Gaussian measurement noise to have a diagonal covariance matrix. Future work consists of extending the approach to Gaussian noise with 


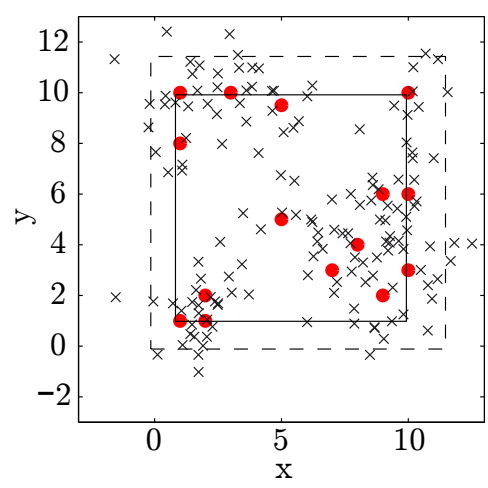

(a) Time step $k=10$.

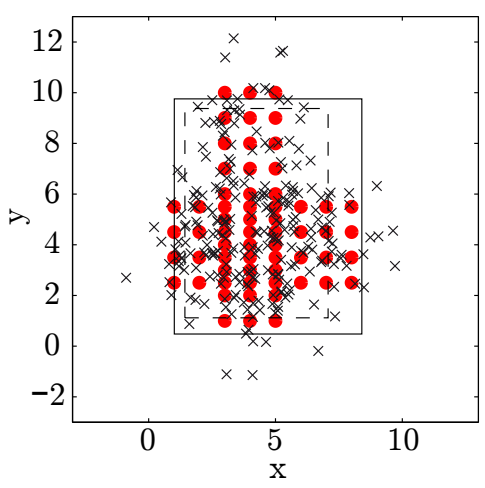

(b) Time step $k=4$.



(c) Time step $k=4$.

Fig. 4: Example: Estimating the minimum bounding rectangle of a fixed set of measurement sources. Measurement sources (red dots) and measurements (blue crosses). The results of the novel estimator is given by the black rectangle, the dashed rectangle is the estimation results for the spatial distribution model. The prior for the rectangle parameters is given by a Gaussian distribution with mean $[-1,9,0,9]^{T}$ and covariance matrix $\operatorname{diag}(4,4,4,4)$.

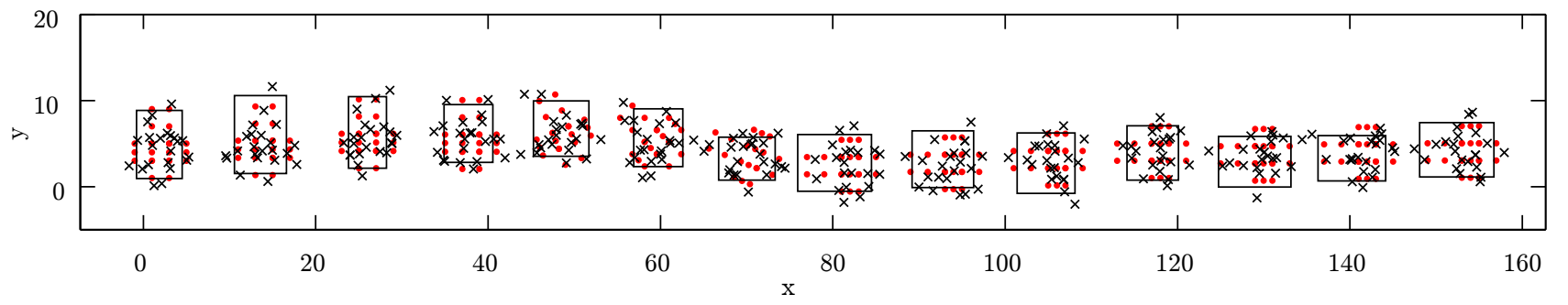

Fig. 5: Tracking a group of point targets: Point targets (red dots), measurements (crosses), and estimated ellipse (red) plotted for several time steps.

non-diagonal covariance matrix. Finally, it will be investigated whether the approach can be extended to other shapes, such as arbitrary oriented rectangles, circles or ellipses. Especially for tracking applications, information about the target orientation is important.

\section{REFERENCES}

[1] D. Hall and J. Llinas, Handbook of Multisensor Data Fusion. CRC Press, May 2001.

[2] M. J. Waxman and O. E. Drummond, "A Bibliography of Cluster (Group) Tracking," Signal and Data Processing of Small Targets 2004, vol. 5428, no. 1, pp. 551-560, 2004.

[3] K. Gilholm and D. Salmond, "Spatial Distribution Model for Tracking Extended Objects," Radar, Sonar and Navigation, IEE Proceedings, vol. 152, no. 5, pp. 364-371, October 2005.

[4] M. Baum and U. D. Hanebeck, "Random Hypersurface Models for Extended Object Tracking," in Proceedings of the 9th IEEE International Symposium on Signal Processing and Information Technology (ISSPIT 2009), Ajman, United Arab Emirates, Dec. 2009.

[5] K. Gilholm, S. Godsill, S. Maskell, and D. Salmond, "Poisson Models for Extended Target and Group Tracking," in SPIE: Signal and Data Processing of Small Targets, 2005.

[6] M. Bensic and K. Sabo, "Border Estimation of a Two-Dimensional Uniform Distribution if Data are Measured with Additive Error," Statistics - A Journal of Theoretical and Applied Statistics, vol. 41, pp. 311-319(9), August 2007.

[7] M. Baum and U. D. Hanebeck, "Tracking an Extended Object Modeled as an Axis-Aligned Rectangle," in 4th German Workshop on Sensor Data Fusion: Trends, Solutions, Applications (SDF 2009), Lübeck, Germany, October 2009.
[8] J. Vermaak, N. Ikoma, and S. Godsill, "Extended object tracking using particle techniques," Aerospace Conference, 2004. Proceedings. 2004 IEEE, vol. 3, pp. -1885 Vol.3, March 2004.

[9] —, "Sequential Monte Carlo Framework for Extended Object Tracking," IEE Proceedings on Radar, Sonar and Navigation, vol. 152, no. 5, pp. 353 - 363, October 2005.

[10] N. Ikoma and S. Godsill, "Extended Object Tracking with Unknown Association, Missing Observations, and Clutter using Particle Filters," in IEEE Workshop on Statistical Signal Processing, 2003, pp. $502-$ 505 .

[11] Z. Zhang, "Parameter Estimation Techniques: A Tutorial with Application to Conic Fitting," Image and Vision Computing, vol. 15, no. 1, pp. $59-76,1997$.

[12] J. Porrill, "Fitting Ellipses and Predicting Confidence Envelopes using a Bias Corrected Kalman filter," Image Vision Comput., vol. 8, no. 1, pp. 37-41, 1990.

[13] T. Ellis, A. Abbood, and B. Brillault, "Ellipse detection and matching with uncertainty," Image Vision Comput., vol. 10, no. 5, pp. 271-276, 1992.

[14] L. de Haan and A. Ferreira, Extreme Value Theory - An Introduction. Springer Verlag, 2006.

[15] P. Embrechts, "Extreme Value Theory: Potential And Limitations As An Integrated Risk Management Tool," Derivatives Use, Trading \& Regulation, vol. 6, 2000.

[16] P. Embrechts, T. Mikosch, and C. Klüppelberg, Modelling Extremal Events: For Insurance and Finance. London, UK: Springer, 1997.

[17] D. Simon, Optimal State Estimation: Kalman, H Infinity, and Nonlinear Approaches, 1st ed. Wiley \& Sons, August 2006.

[18] J. Kotecha and P. Djuric, "Gaussian Particle Filtering," IEEE Transactions on Signal Processing, vol. 51, no. 10, pp. 2592 - 2601, Oct. 2003. 